\title{
The impact on education of Astronomical Olympiads and the International Olympiad on Astronomy and Astrophysics
}

\author{
Greg Stachowski ${ }^{1,3}$, and Aniket Sule ${ }^{2,3}$ \\ ${ }^{1}$ Pedagogical University of Cracow, Podchorazych 2, 30-084 Krakow, Poland \\ ${ }^{2}$ Homi Bhabha Centre for Science Education, VN Purav Marg, Mankhurd, 400088 Mumbai, India \\ ${ }^{3}$ International Olympiad on Astronomy and Astrophysics
}

\begin{abstract}
Astronomical Olympiads and similar competitions for highschool students have been run in some countries for more than half a century, and last year marked the tenth anniversary of the largest such competition with global reach, the International Olympiad on Astronomy and Astrophysics. The effect of these has been to reach out to a large number of school students who might not otherwise have considered astronomy as a subject; help maintain a high, guided standard of astronomy education even in countries where astronomy is not (or no longer) on the curriculum; and to encourage those students who participate to strive harder and pursue astronomy further by giving them goals to aim for, rewarding their efforts with medals, recognition and participation in the international events in interesting locations and, above all, showing them that there are many other students just like them both in their own country and around the world. Many of the students go on to careers in astronomy education or research. We believe that Astronomy Olympiads are a very valuable element in the astronomy education framework which can be used to further the common goal of sustaining and growing the astronomical community.
\end{abstract}

\section{Introduction}

In many countries, 'olympiads' are a form of extra-curricular competition for senior high school students ( $\sim 15-19$ years of age) testing their knowledge and skills in various academic subjects, including the sciences, mathematics, computing and languages. Often broader in scope than the usual school syllabus for these subjects, they typically include questions designed to test the students reasoning abilities as much as their knowledge and rounds of practical as well as theoretical tests. At the national level, there may be multiple rounds or division into regional or age groups, culminating in a final resulting in the award

1 Corresponding author: greg@astro.as.up.krakow.pl 
of medals and other prizes. Good results may also provide other privileges, such as exemption from university entrance exams or credits towards a scholarship.

Similar competitions are organised annually on the regional and international level. The sciences are collectively represented by the International Science Olympiads, also including mathematics, philosophy and mathematical linguistics. Two events are dedicated to astronomy: the International Astronomical Olympiad (founded in 1996) and the International Olympiad on Astronomy and Astrophysics (founded in 2007). The latter, much larger, event is the focus of this article.

\section{The International Olympiad on Astronomy and Astrophysics}

The International Olympiad on Astronomy and Astrophysics (or IOAA) arose from discussions which took place at the 2006 International Physics Olympiad (IPhO) where the need was expressed for an independent event based on the form, democratic governance structure and statutes of the IPhO but tailored to the specific topics and skills encountered in modern astronomy and astrophysics. Since the initial event where 22 countries took part the IOAA has grown regularly and as of 2017 over 40 countries from every continent participate annually.

Table 1. Previous International Olympiads on Astronomy and Astrophysics events

\begin{tabular}{|l|l|}
\hline Year & Location \\
\hline 2007 & Chiang Mai, Thailand \\
\hline 2008 & Bandung, Indonesia \\
\hline 2009 & Tehran, Iran \\
\hline 2010 & Beijing, China \\
\hline 2011 & Krakow, Poland \\
\hline 2012 & Rio de Janeiro \& Vassouras, Brazil \\
\hline 2013 & Volos, Greece \\
\hline 2014 & Suceava, Romania \\
\hline 2015 & Magelang, Indonesia \\
\hline 2016 & Bhubaneswar, India \\
\hline 2017 & Phuket, Thailand \\
\hline
\end{tabular}

\subsection{Participation}

Student participants must be under the age of 20 and must be either in high-school or have completed it the year of the competition. Each country sends a team consisting of up to five students and up to two 'team leaders'. Team selection is up to the individual countries but typically involves a competitive process, such as the national astronomical olympiad. Guest teams and observers may also be invited by the hosts at their discretion but must be identified as such. The team leaders together form the International Board which oversees the testing process and makes decisions through a democratic process overseen by an elected President and General Secretary. Students are isolated from the team leaders and the 
internet and mobile communications before either group sees the questions and until the examinations are complete (during this time they are looked after by local guides)

\subsection{Format}

Each event takes place over 10 days, and includes three rounds of individual competition: theoretical problem solving (contributing $50 \%$ of the final mark for each participant), data analysis $(25 \%)$ and night sky observation $(25 \%)$.

The theoretical round includes short and long examination-type questions usually involving physical and astrophysical formulae, derivation and calculation. The syllabus is the same as any typical first-year undergraduate curriculum for astronomy and astrophysics. The problems are designed such that calculus is not required for the model solution (due to differences in the school syllabus in various countries). The student may use any valid method for their solution (including calculus if they know it), and indeed during marking it is common to find problems solved in several different ways. A data sheet gives standard constants and any formulae not expected to be known at the high-school level are given and explained in the question. Questions which teach as well as test are preferred, often drawing from recent results in astrophysics or presenting a problem in a way which might not have been encountered at school, so that the student walks away with some new knowledge or insight.

Together the data analysis and night-sky observations are designed to represent the practical aspects of modern astronomy. The data analysis test uses real data and requires the student to draw inferences from it, using simple statistics and including an awareness of uncertainties. Past examples include aperture photometry on a CCD image, measurement of galaxy rotation on a spectrum, and analysis of cepheid brightnesses. The observational part involves naked eye and telescopic observation and may include both the real sky and planetarium simulations. Both identification of night-sky objects and more quantitative observations are assessed, and the students are allowed to familiarise themselves with any equipment beforehand.

A group competition is also often organised, the format of which has so far varied between events, encouraging cooperation between the students in line with the way most real science is carried out. (An interesting feature of this competition is that mixed teams from different countries are allowed and have proved very popular with the students.)

In between the examinations the students participate in excursions and cultural events, with the intention of introducing them to the country and people hosting the event. For many students this is the first time they have been abroad, not to mention visiting a country far across the world. The host country covers all costs except for travel to and from the event. At this time there is no participation fee for 'main' teams.

\subsection{Question-setting and assessment}

An initial set of questions (including a number of 'spares') for each part of the competition is prepared by the local organisers, and discussed and amended as necessary by the International Board. Once agreed upon, the questions are translated by the team leaders into their national languages, as the students are not required to understand English (Fig 1). The questions are designed such that answers require minimal exposition by the student to facilitate marking. Marks are given for correct work, not just a numerical value, and significant figures and units are also assessed. Once the examinations are complete, each student's answers are assessed independently by both a local jury and the team leaders, and any discrepancies are resolved in a moderation meeting before the final results are published. 
Gold, silver and bronze medals and honourable mention certificates are awarded in a blind process based on normalised scores. Between $5-10 \%$ of participants will achieve scores high enough for a gold medal, with proportionately higher numbers of the other medals. Individual prizes for the single highest score in each section are also given.

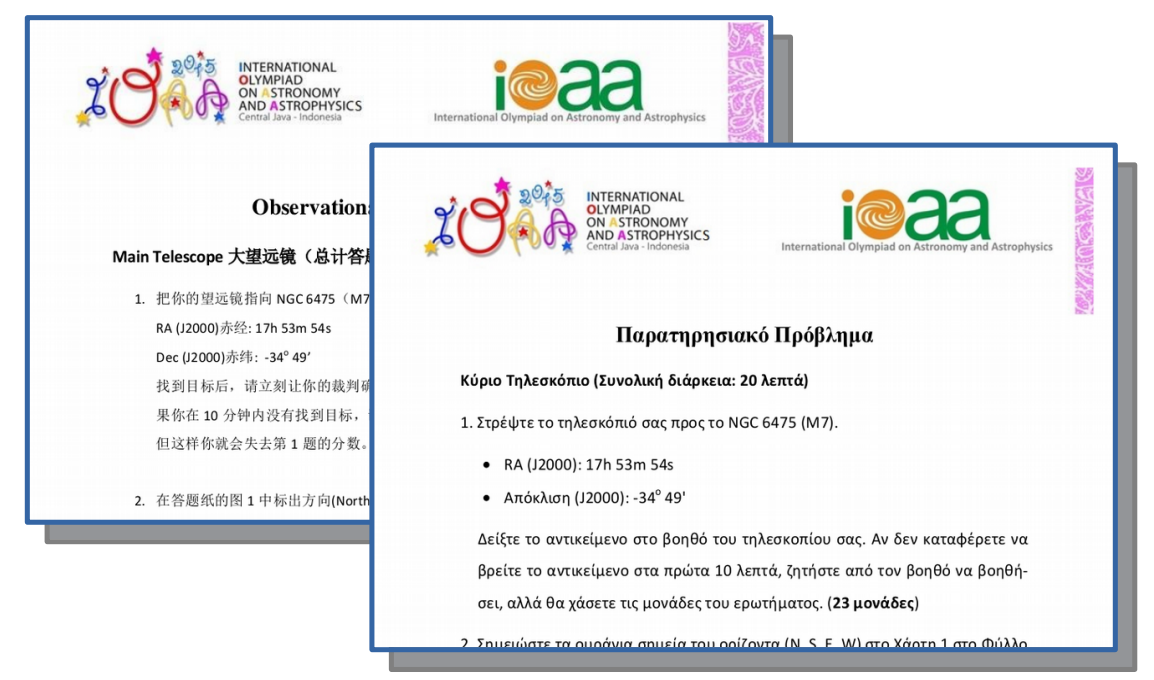

Fig. 1. Illustration of papers in different languages (from the 2015 IOAA).

\section{Impact}

We have found that opportunity to visit other countries as part of the team and the positive experiences related by past participants is a motivating factor for school students who are looking for a challenge but who might not have otherwise considered astronomy, and has drawn increasing numbers to national competitions. Furthermore the IOAA helps to maintain the standard of astronomy education in countries where astronomy is not on the curriculum by providing syllabus, past papers etc., and providing tangible goals for the students to aim for (medals, recognition, travel) when learning about astronomy. Perhaps most importantly, participation shows students who may have been the only ones in their village or town 'into astronomy' that there are many others just like them both in their own country and around the world.

The desire to take part in the IOAA has resulted in the startup of national astronomical olympiads in countries where students who had heard of the IOAA wanted to participate but no local competition mechanism existed (e.g. Bangladesh, Mali, Malaysia, Singapore, UK). This has in turn increased the availability of astronomy education in these countries.

Most participating countries organise a residential training and selection program for the team or, frequently, a larger group. This may last from a few days (e.g. Bangladesh, Poland) to a few weeks (e.g. Iran). The questions in both national selection tests and IOAA past papers become informal standards for teachers and future students. The IOAA has also published a book of past problems which includes model solutions[1]. This provides a valuable teaching resource in countries where astronomical textbooks are in short supply. 
Through careful question design and (where possible) lectures and excursions, each IOAA also introduces students to the latest developments in astronomy and astrophysics in such a way that they can understand the key science at their level. Students also meet professional astronomers from both their own and other countries, as the team leaders are in the majority selected from university staff, as well as astronomical societies, observatories and planetariums (perhaps surprisingly, school teachers make up only about 1/8 of the team leaders).

Inspired by this experience many students go on to careers in astronomy education and research with notable achievements (e.g. the first author of [2] is a previous gold medallist and winner of the Olympiad). The additional study involved in preparing for the IOAA serves them well during the first years of their undergraduate studies, putting them ahead of their peers. Moreover, in recent years past participants have also begun returning as team leaders showing that the event is self-sustaining. Previous participants have also 'exported' the Olympiad to other countries, for example when moving abroad to countries which had not participated for work or PhD studies (Canada and the UK are prominent examples). Thus the IOAA has become a positive force for promoting astronomy education and 'pulling in' the next generation of professional astronomers and educators from around the globe.

Finally the organisation of an IOAA event also has an impact on local education in the host country, with equipment from the IOAA (e.g. telescopes used during the competition) being passed on to schools, local volunteers being trained in astronomy, the involvement of local amateur astronomers in the night-sky observing round, and the involvement of language students as guides for the various national teams.

\section{Conclusions}

National and regional astronomical olympiads and the International Olympiad on Astronomy and Astrophysics form a structured framework of events capable of reaching out to and motivating high-school students to study astronomy and astrophysics, rewarding them at each step. This is especially important with astronomy not being well-represented in the high-school syllabus in many countries, and in a common social environment where competition and achievement are often valued by both students and parents over 'learning for its own sake'.

The authors have already seen anecdotal evidence that many next generation astronomers would come through this channel, and would like to encourage teachers and astronomy educators to use the IOAA as a motivator and to consider preparing students for participation, particularly in those countries which do not yet take part (particularly we would like to see participation from the remaining countries which already have national olympiads in astronomy). We would also encourage professional astronomers from participating countries to contribute their expertise to their national programs where possible.

The authors of this article are, respectively, the current President and General Secretary of the IOAA. 


\section{References}

1. A. Sule, International Olympiads on Astronomy and Astrophysics - Problems and Solutions (Orient Blackswan, 2015, ISBN 978-8173719806)

2. P. Mróz, A. Udalski, P. Pietrukowicz, M. K. Szymański, I. Soszyński, Ł. Wyrzykowski, R. Poleski, S. Kozłowski, J. Skowron, K. Ulaczyk, D. Skowron, M. Pawlak Nature 537, 649 (2016) 